\title{
Otorhinolaryngological Manifestations in COVID-19 Patients
}

\author{
Rukmini M Prabhu ${ }^{1}$, Kartik Irappa Patil ${ }^{1 *}$, Shouab Mohammed ${ }^{2}$, Shivaranjini S ${ }^{2}$ and Borlingegowda Viswanatha ${ }^{3}$ \\ ${ }^{1}$ Senior Residents, Department of ENT, Bangalore Medical College\& Research Institute, India \\ ${ }^{2}$ Postgraduates, Department of ENT, Bangalore Medical College\& Research Institute, India \\ ${ }^{3}$ Professor and Head, Department of ENT, Bangalore Medical College\& Research Institute, India
}

*Corresponding author: Kartik Irappa Patil, Senior Resident, Department of ENT, Bangalore Medical College\& Research Institute, Bangalore, India

\begin{abstract}
Introduction:The World Health Organization confirmed COVID 19 as a pandemic on 11th March 2020. Though the most common symptoms of COVID-19 are fever, cough, myalgia, fatigue, and difficulty breathing, ear, nose, and throat (ENT) symptoms, including loss of sense of smell and/or loss of sense of taste have been reported as symptoms caused by the virus.

Aims and Objectives:To detect and discuss the different otorhinolaryngology (ORL) manifestations reported in COVID-19 positive patients.

Materialsand Methods: This observational study was done on 2000 patients with a positive COVID 19 test admitted to a tertiary care hospital. The patients were assessed for their signs and symptoms and the findings were analysed.

Results: The patients ranged in age from 18 to 70 years, including 1090 males (54.5\%) and 910 females (45.5\%). The most common presenting symptom was fever seen in 1500 (75\%) patients. Other symptoms included cough in 1100 (55\%) patients, expectoration seen in $446(22.3 \%)$, breathlessness in 302(15.1\%), nausea and vomiting in 80(4\%) patients, fatigue/malaise in 568 $(28.4 \%)$ patients.The most common otorhinolaryngological manifestation were sore throat seen in $460(23 \%)$ patients, running nose in $292(14.6 \%)$, olfactory disturbances in 180 (9\%), nasal obstruction in 156(7.8\%), headache in 222(11.1\%), pharyngeal congestion in $208(10.4 \%)$, tonsillar hypertrophy seen in $62(3.1 \%)$ patients.

Conclusion: Though, the most common presenting symptoms in COVID-19 patients are fever and cough, a significant proportion of patients does have ENT manifestations which may go unnoticed. Hence it becomes necessary to screen all COVID patients for ENT symptoms so that high quality care can be provided for patients.This also facilitates care givers to take necessary precautions to protect themselves.
\end{abstract}

Keywords: COVID 19; otorhinolaryngological; manifestations

\section{Introduction}

The Severe Acute Respiratory Syndrome Coronavirus 2 (SARSCoV-2), firstly known as the 2019 novel Coronavirus (2019-nCoV), started in Wuhan in China in December 2019[1].The World Health Organization confirmed it as a pandemic on 11thMarch 2020[2]. Although this virus can cause severe respiratory failure and even death in infected patients, it has spread rapidly and continues to spread among people because it can cause mild or no symptoms in the majority of cases[3,4].The most common symptoms of COVID-19 are fever, cough, myalgia, fatigue, and difficulty breathing. In addition, ear, nose, and throat (ENT) symptoms, including loss of sense of smell and/or loss of sense of taste have been reported as symptoms caused by the virus.The aim of this study was to detect and discuss the different otorhinolaryngology (ORL) manifestations reported in COVID-19 positive patients. 


\section{Materials and Methods}

All patients with confirmed reverse transcriptase polymerase chain reaction (RT-PCR)-positive testing for the SARS -CoV-2 viral genome were assessed for symptoms and signs. 2000 patients who presented to a tertiary care designated COVID hospital were included in the study. Health workers with confirmed positive PCR test results were voluntarily enrolled in the study. All subjects provided informed consent to participation in the study.

\section{The inclusion criteria were}

a) Patients $>18$ years of age with a positive COVID -19 test.

b) Patients having mild to moderate symptoms.

\section{The exclusion criteria were}

a) Age $<18$ years old.

b) No confirmed positive PCR test result.

c) A history of chronic nasal problems.

d) Recent head injury, recent nasal surgery.

e) Severe respiratory failure or treatment in the intensive care unit.

\section{Results}

A total of 2000 patients were included in the study. The patients ranged in age from 18 to 70 years, including 1090 males (54.5\%) and 910 females (45.5\%)(Figure 1\& Table 1).The most prevalent comorbidities in these patients were (Figure $2 \&$ Table 2).

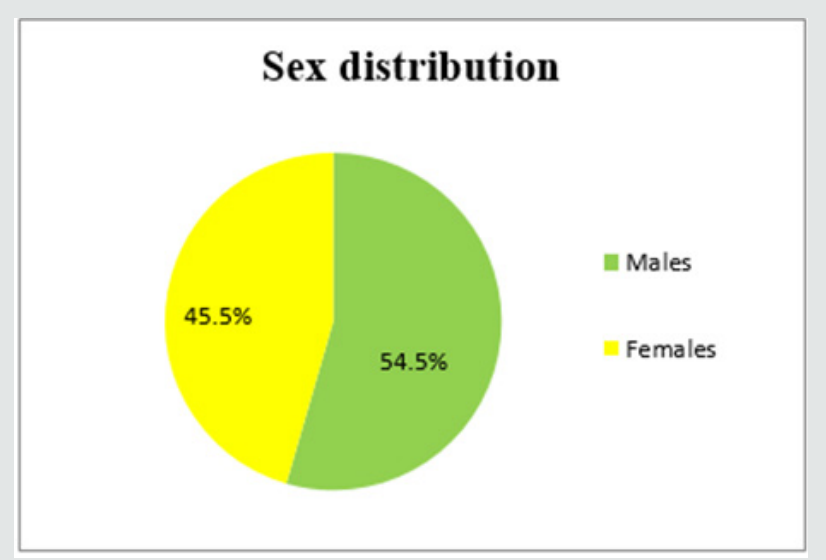

Figure 1: Showing the sex distribution of patients.

Table 1: Showing the sex distribution of patients.

\begin{tabular}{|c|c|c|}
\hline & No. of Patients & Percentage\% \\
\hline Male & 1090 & 54.5 \\
\hline Female & 910 & 45.5 \\
\hline
\end{tabular}

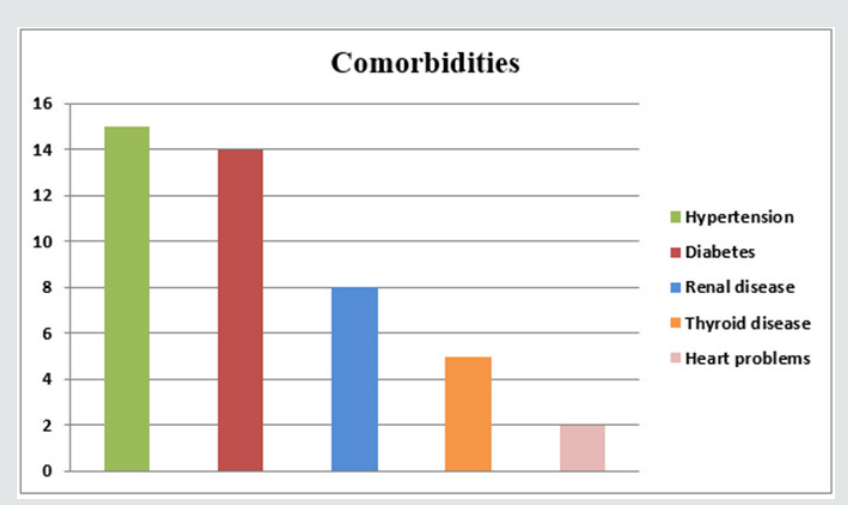

Figure 2: Showing various comorbidities seen in patients.

Table 2: Showing comorbidities seen in patients.

\begin{tabular}{|c|c|c|}
\hline Comorbidities & No. of Patients(n=2000) & Percentage \% \\
\hline Hypertension & 300 & 15 \\
\hline Diabetes & 280 & 14 \\
\hline Renal disease & 160 & 8 \\
\hline Thyroid disease & 100 & 5 \\
\hline Heart problems & 40 & 2 \\
\hline Total & 880 & 44 \\
\hline
\end{tabular}
a) High blood pressure in 15\%,
b) Diabetes in $14 \%$,
c) Renal disease in $8 \%$,
d) Thyroid diseases in 5\%,
e) Heart problems in $2 \%$.

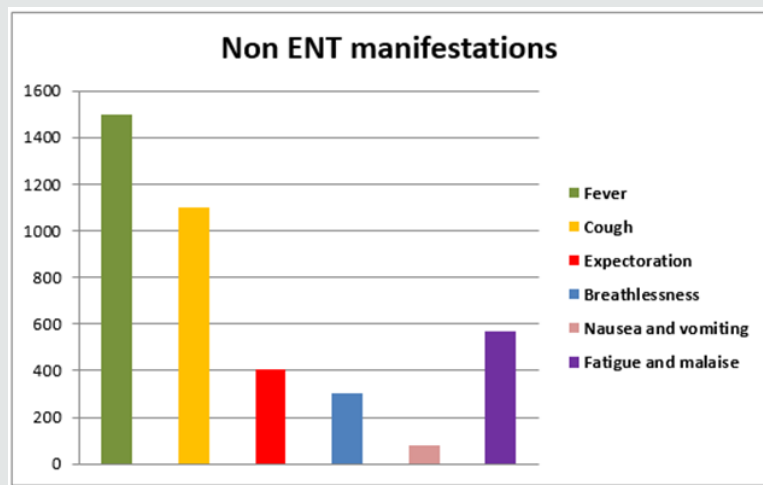

Figure 3: Showing various Non-ENT manifestations in patients.

The most common presenting symptom was fever seen in $1500(75 \%)$ patients. Other symptoms included cough in 1100 (55\%) patients, expectoration seen in 446 (22.3\%), breathlessness in $302(15.1 \%)$, nausea and vomiting in $80(4 \%)$ patients, fatigue/ 
malaise in 568 (28.4\%) patients (Figure 3\& Table 3).Other rare symptoms were hemoptysis, chest pain, constipation and diarrhoea. The most common otorhinolaryngological manifestation was sore throat seen in 460 (23\%) patients. Other manifestations include running nose in 292 (14.6\%), olfactory disturbances in 180 (9\%), nasal obstruction in $156(7.8 \%)$ patients, headache in $222(11.1 \%)$, pharyngeal congestion in 208(10.4\%), tonsillar hypertrophy seen in $62(3.1 \%)$ patients. Dizziness was seen in $5(0.25 \%)$ patients. Epistaxis was seen in $8(0.4 \%)$ patients (Figure $4 \&$ Table 4$).$ No patients reported facial edema or tenderness, diminution of hearing, hoarseness, or stridor.

Table 3: Showing non-ENT manifestations in patients.

\begin{tabular}{|c|c|c|}
\hline Symptoms & No.of Patients $(\mathbf{n = 2 0 0 0 )}$ & Percentage(\%) \\
\hline Fever & 1500 & 75 \\
\hline Cough & 1100 & 55 \\
\hline Expectoration & 446 & 22.3 \\
\hline Breathlessness & 302 & 15.1 \\
\hline $\begin{array}{c}\text { Nausea and } \\
\text { vomiting }\end{array}$ & 80 & 4 \\
\hline Fatigue and malaise & 568 & 28.4 \\
\hline
\end{tabular}

\section{ENT manifestations}

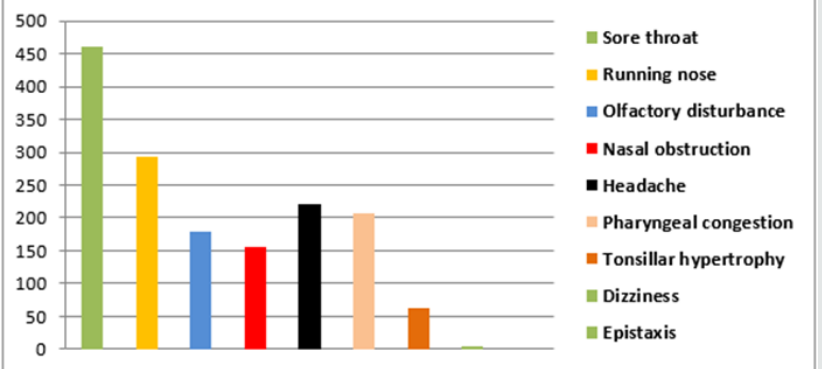

Figure 4: Showing various ENT manifestations in patients.

Table 4: Showing non-ENT manifestations in patients.

\begin{tabular}{|c|c|c|}
\hline $\begin{array}{c}\text { Symptoms and } \\
\text { Signs }\end{array}$ & No.of patients (n=2000) & Percentage (\%) \\
\hline Sore throat & 460 & 23 \\
\hline Running nose & 292 & 16.6 \\
\hline $\begin{array}{c}\text { Olfactory } \\
\text { dysfunction }\end{array}$ & 180 & 9 \\
\hline Nasal obstruction & 156 & 7.8 \\
\hline Headache & 222 & 11.1 \\
\hline $\begin{array}{c}\text { Pharyngeal } \\
\text { congestion }\end{array}$ & 202 & 10.4 \\
\hline $\begin{array}{c}\text { Tonsillar } \\
\text { hypertrophy }\end{array}$ & 62 & 3.1 \\
\hline Dizziness & 5 & 0.25 \\
\hline Epistaxis & 8 & 0.4 \\
\hline
\end{tabular}

\section{Discussion}

In December 2019, a novel coronavirus epidemic, caused by the severe acute respiratory syndrome coronavirus-2 (SARS-CoV-2) emerged from China[3]. The disease is widely distributed, making it an important pathogen with an unrestricted health threat [7]. The nasal, nasopharyngeal and/or the oropharyngeal tissue is one of the main harbor sites of the infection, main site of taking the sample for testing and a main source of transmission of infection. However, most published COVID-19 researches are focused on the lower respiratory tract manifestation and sequels due to their life-threatening nature.The literature that is available on ENT manifestation during COVID-19 infection is still sparse, thus, there is value in studying ENT manifestations of such a novel virus[8]. In our study, we have tried to collect the data concerning the ENT manifestations in the laboratory confirmed COVID-19 cases that were mild to moderately symptomatic.In the systematic review conducted by El Anwar M et al. they observed that fever (reported in $73.5 \%$ of the included patients), and cough (reported in 61\%) are the dominant symptoms ofCOVID-19[8]. The findings of our study were similar with fever being seen in $75 \%$ of the patients and cough being seen in $55 \%$ of the patients. However, in the study by Salepci E et al, the most common general symptom was fatigue $(71.3 \%)$ followed by cough (54.3\%), and fever (50.7\%)[9].

Lovato et al. criticizing the largest meta-analysis study on COVID-19 to date by Sun et al, suggested that true prevalence of sore throat was underestimated[10].In a followup systemic review, Lovato et al. reported that $12.4 \%$ of patients had sore throat[11]. In our study, $23 \%$ of the patients suffered from sore throat. In the study by Salepci et al, the prevalence of sore throat was $26 \%$ [9].In the review by El Anwar et al, the most common ENT manifestations for COVID-19 were sore throat (11.3\%) and headache(10.7\%).They concluded that their incidence is much less than the incidence of fever and cough in COVID-19 patients[8]. In the study conducted by Sakalli E et al.the most frequent ENT-related symptoms of the patients were nasal obstruction (53.5\%), loss of sense of smell (51.2\%), sore throat (50.2\%), loss of sense of taste $(47.1 \%)$, and rhinorrhea (38.5\%) [12]. In our study the most common otorhinolaryngological manifestation was sore throat seen in 460 (23\%) patients. Other manifestations include running nose in 292 (14.6\%), nasal obstruction in 156(7.8\%) patients, headache in 222(11.1\%), pharyngeal congestion in 208(10.4\%), tonsillar hypertrophy seen in $62(3.1 \%)$ patients. In the review by El Anwar $\mathrm{M}$ et al, no emergency ENT symptoms as bleeding per nose or throat or stridor were seen [8]. In our study, 8 patients presented with epistaxis, but there were no cases of stridor or upper airway obstruction.Varia et al.performed objective smell and taste tests were performed on 72 patients with positive PCR for COVID-19 and with no previous history of smell and taste dysfunction.They found that $73.6 \%$ of patients had smell or taste dysfunction during 
the course of the COVID-19 with $14.4 \%$ having isolated olfactory dysfunctions [13]. Sakalli Eet al, in their study concluded that loss of sense of smell/ taste is a common symptom in COVID-19 and may be the first and/or only symptom of this disease. In our study, $9 \%$ of the patients presented with olfactory disturbance.

In the study conducted by Salepci et al, out of 223 patients, only five patients stated that they had new onset complaints of dizziness and two that of hearing loss[9].They proposed that these complaints might not be related to COVID-19, but simply coincide with disease onset. Auditory manifestation was not reported in the studies on COVID-19 and auditory complication due to coronavirus is little mentioned in the literature[8].In our study, 5(0.25\%) patients presented with dizziness, and no patients complained of hearing loss. Otorhinolaryngologic examinations produce aerosol, hence it is important to determine which patients are at higher risk of disease transmission and take the necessary precautions[14,15]. Salepci et al. observed that relying solely on fever measurement and questioning of patients only for general symptoms creates false confidence. They advocated that Otorhinolaryngologist should be alert not only for general symptoms but also for otorhinolaryngologic symptoms that could be associated with COVID-19. The absence of common symptoms must not be interpreted as the absence of the disease[9].

\section{Conclusion}

Though, the most common presenting symptoms in COVID-19 patients are fever and cough, a significant proportion of patients does have ENT manifestations which may go unnoticed. Hence it becomes necessary to screen all COVID patients for ENT symptoms so that high quality care can be provided for patients.This also facilitates care givers to take necessary precautions to protect themselves.

\section{References}

1. Xia W, Shao J , Guo Y, Peng X, Li Z, et al. (2020) Clinical and CT featuresin pediatric patients with COVID-19 infection: different points from adults. Pediatr Pulmonol 55(5): 1169-1174.
2. Cucinotta D, Vanelli M (2020) WHO declares COVID-19 a pandemic? Actabio-medica: Atenei Parmensis 91(1): 157-160.

3. Guan WJ, Ni ZY, Hu Y (2020) Clinical characteristics of coronavirus disease 2019 in China. N Engl J Med 382(18): 1708 -1720.

4. Wu Z, McGoogan J (2020) Characteristics of and Important Lessons from the Coronavirus Disease 2019 (COVID-19) Outbreak in China. JAMA 323(13): 1239-1242.

5. Chen N, Zhou M, Dong X (2020) Epidemiological and clinical characteristics of 99 cases of 2019 novel coronavirus pneumonia in Wuhan, China: a descriptive study. Lancet 395(10223): 507-513.

6. Wu YC, Chen CS, Chan YJ (2020) Overview of the novel coronavirus (2019 -nCoV): The pathogen of severe specific contagious pneumonia (SSCP). J Chin Med Assoc.

7. Hassan SA, Sheikh FN, Jamal S, Ezeh JK, Akhtar A (2020) Coronavirus(COVID-19): a review of clinical features, diagnosis, and treatment. Cureus 12(3): e7355.

8. El.Anwar M, Elzayat S, Fouad Y (2020) ENT manifestation in COVID 19 patients. Auris Nasus Larynx 47(4): 559-564.

9. Salepci, E., Turk, B., Ozcan, S.N. et al. Symptomatology of COVID-19 from the otorhinolaryngology perspective: a survey of 223 SARS-CoV-2 RNApositive patients. Eur Arch Otorhinolaryngol (2020).

10. Sun P, Qie S, Liu Z (2020) Clinical characteristics of hospitalized patients with SARS-CoV-2 infection: a single arm meta-analysis. J Med Virol 92: 612-617.

11. Lovato A, Rossettini G, Filippis C (2020) Sore throat in COVID-19: Comment on "Clinical characteristics of hospitalized patients with SARSCoV-2 infection: A single arm meta-analysis". Journal of Medical Virology 92(7): 714-715.

12. Sakalli E, Temirbekov D, Bayri E, Alis E, Erdurak S, et al. (2020) Ear nose throat-related symptoms with a focus on loss of smell and/or taste in COVID-19 patients. American Journal of Otolaryngology 41(6): 102622.

13. Vaira L, Deiana G, Fois A, Pirina P, Madeddu G, et al. (2020) Objective evaluation of anosmia and ageusia in COVID -19 patients: Single-center experience on 72 cases. Head \& Neck 42(6): 1252-1258.

14. Shuman A (2020) Navigating the Ethics of COVID-19 in Otolaryngology. Otolaryngology-Head and Neck Surgery 162(6): 811-812.

15. MeccarielloG, Gallo O (2020) What ENT doctors should know about COVID-19 contagion risks. Head Neck 42: 1248-1249.
This work is licensed under Creative Commons Attribution 4.0 License

To Submit Your Article Click Here: Submit Article

DOI: $10.32474 /$ SJO.2020.05.000212

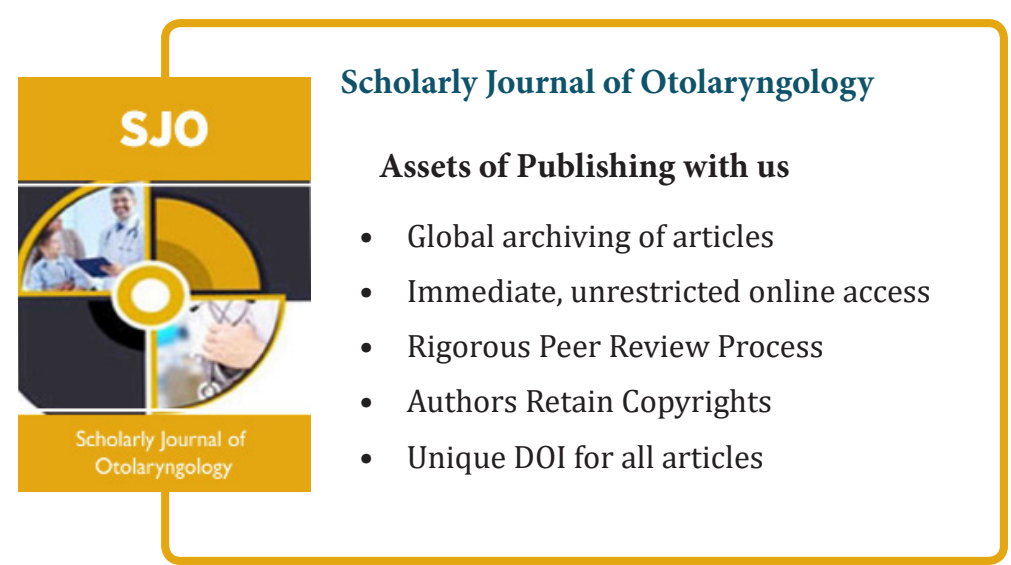

Citation: Rukmini M Prabhu, Kartik Irappa Patil, Shouab Mohammed, Shivaranjini S, Borlingegowda Viswanatha. Otorhinolaryngological 